\title{
Delayed formation of sterile abscess after zygomaticomaxillary complex fracture treatment with bioabsorbable plates
}

\author{
GyeongHyeon Doh', \\ Sujin Bahk ${ }^{1}$, \\ Ki Yong Hong ${ }^{1}$, \\ SooA Lim ${ }^{1}$, \\ Kang Min $\mathrm{Han}^{2}$, \\ SuRak Eo ${ }^{1}$ \\ Departments of ${ }^{1}$ Plastic and Reconstructive \\ Surgery and ${ }^{2}$ Pathology, Dongguk University \\ Ilsan Hospital, Goyang, Korea
}

\begin{abstract}
We present a patient who showed a sterile abscess after facial bone fixation with bioabsorbable plates and screws. He had zygomaticomaxillary complex and periorbital fracture due to falling down. The displaced bones were treated by open reduction and internal fixation successfully using bioabsorbable plate system. However, at postoperative 11 months, abrupt painless swelling was noted on the previous operation sites, left lateral eyebrow and lower eyelid. By surgical exploration, pus-like discharge and degraded materials were observed and debrided. The pathologic analysis revealed foreign body reaction with sterile abscess. This complication followed by bioabsorbable device implantation on maxillofacial bone surgery has been rarely reported in which we call attention to the maxillofacial plastic surgeons.
\end{abstract}

Keywords: Sterile abscess / Foreign body reaction / Bioabsorbable implants

\section{INTRODUCTION}

Bioabsorbable plates and screws are commonly used for bony fixation in various surgical fields including face [1-6] and hand $[7,8]$. In addition to having characteristics of biological degradation and tissue-compatibility, they have reliable advantages over metal implants such as radiolucency, magnetic resonance imaging compatibility and limiting stress shielding [7]. However, these materials have some disadvantages of low strength, rapid loss of initial strength and relatively high refracture rate [8] compared to metal implants. When first generation of bioabsorbable implants consisting mainly of polyglycolic acid were introduced, they had weak mechanical properties and fast degradation rates. Therefore, the stronger implant composed of trimethylene carbonate, L-lactide, and D,L-lactide has been developed to provide improved bony stability after fixation [7-11].

Correspondence: Su Rak Eo

Department of Plastic and Reconstructive Surgery, Dongguk University Ilsan Hospital, 27 Dongguk-ro, Ilsandong-gu, Goyang 10326, Korea

E-mail: sreodoc@gmail.com

Received March 7, 2018 / Revised May 22, 2018 / Accepted June 5, 2018
However, there is another problem that is easy to overlook; lateoccurring reaction such as delayed foreign body reaction or delayed sterile abscess. Previous studies reported that this complication has been mainly noted in shallow regions covered with soft tissue over the plate, such as frontozygomatic suture, orbital rim [1-3], and metacarpal bone [7,8]. We report a case of delayed sterile abscess associated with bioabsorbable plates and screws at postoperative 11 month and remind that plastic surgeons should consider this complication prior to using bioabsorbable materials.

\section{CASE REPORT}

A 39-year-old male had a left zygomaticomaxillary complex fracture. He was treated with bioabsorbable plates and screws (Resorb-x; KLS-Martin, Jacksonville, FL, USA) on the frontozygomatic suture, orbital floor, infraorbital rim, and anterior wall of the maxilla. Resorb-x is a copolymer of poly-DL-lactic acid. It is $1.0 \mathrm{~mm}$ thick and easy to apply because it changes smoothly when reacted to warm water. Furthermore, it is known to maintain mechanical stability over 10 weeks, and continues to absorb at least 
for 72 weeks after implantation [12].

Although the follow-up was uneventful until 11 months postoperatively, the patient visited us with severe painless swelling, which arose 2 months ago abruptly on the previous operation site, left lateral eyebrow region. Physical examination showed $2 \times 2 \mathrm{~cm}$ sized swelling of the soft tissue without tenderness (Fig. 1). Under local anesthesia, an incision was made on the previous surgical site for exploration. A mass and milky pus-like discharge were drained out with scattered white degraded materials (Fig. 2). We

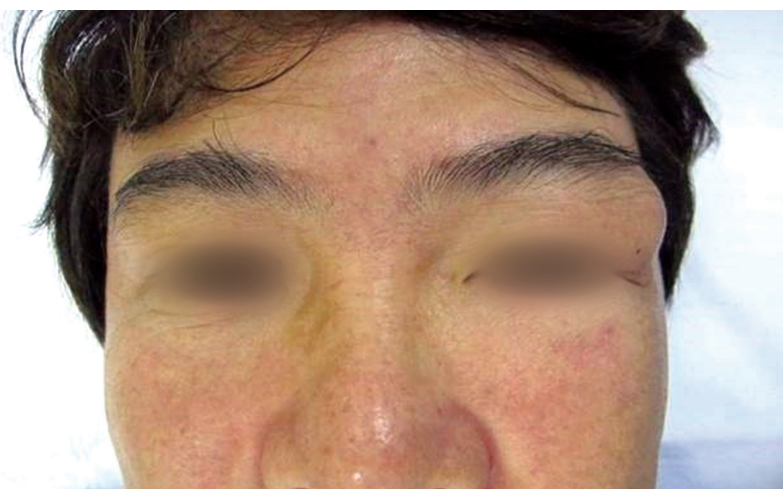

Fig. 1. Postoperative photograph on left lateral eyebrow region in 11 months after open reduction and internal fixation with bioabsorbable plates and screws.

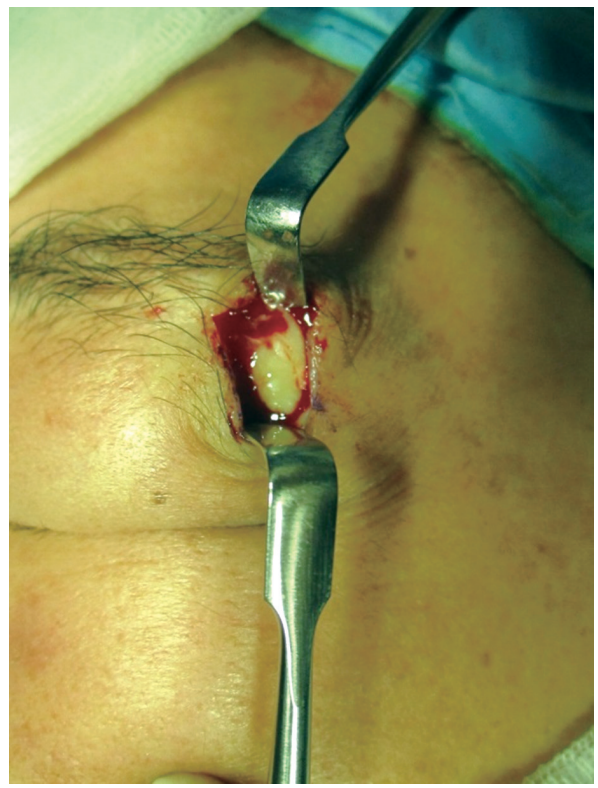

Fig. 2. Intraoperative photograph on left lateral eyebrow region. A mass and milky pus-like discharge were observed with scattered white degraded materials. removed all the unusual tissues and fluids with copious irrigation. The largest white material was $0.3 \mathrm{~cm}$, gray to white, relatively solid and fibrotic. The tissue biopsy for the mass revealed foreign body granuloma (Fig. 3) and wound culture demonstrated no bacterial growth. Just 6 days later, the patient revisited us for the recurred swelling of left lower eyelid, which was the previous surgical site. We approached the lesion under local anesthesia. Suspicious remnants of degraded plates and screws as well as pus-like discharge were detected grossly.

Despite of two explorations, painless local swelling was still persisted on the left lateral eyebrow and lower eyelid region for 10 days (Fig. 4). Therefore, we decided to perform en-bloc resection of the lesion under general anesthesia. Pus-like discharge, whitish granuloma-like mass and remnants of degraded plates were
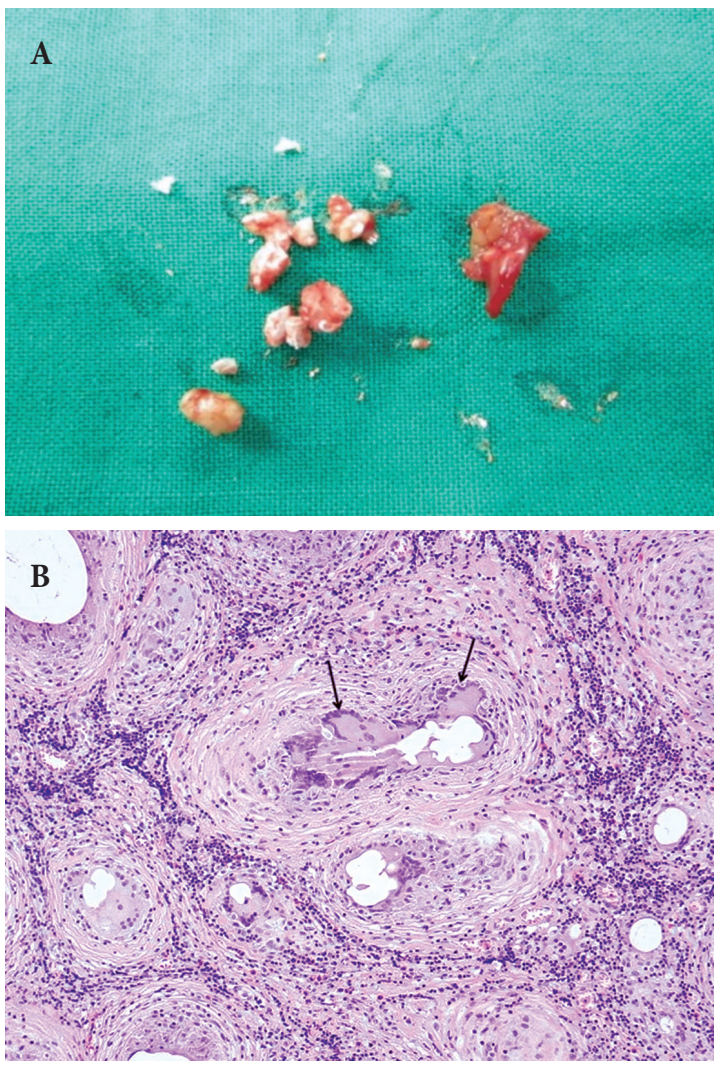

Fig. 3. (A) Specimens from left lateral eyebrow region. The largest white material was relatively solid and fibrotic and measured $0.3 \mathrm{~cm}$. (B) Foreign body granulomas. Each granuloma consists of a central empty space and surrounding inflammatory cells. The inflammatory cells consist of epithelioid histiocytes and multinucleated giant cells (arrows). There are dense lymphocytes and some eosinophils between granulomas $(\mathrm{H} \& \mathrm{E}, \times 100)$. 


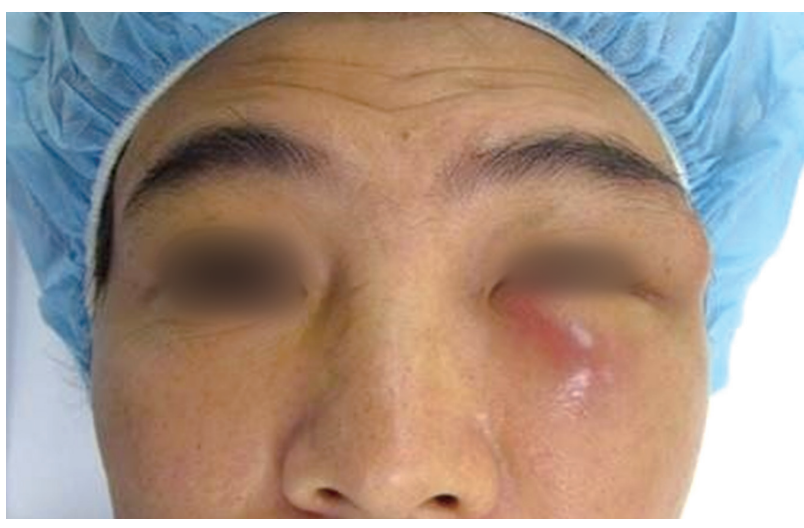

Fig. 4. Preoperative photograph of left periorbital region before enbloc resection of the lesion under general anesthesia.

found deeply around the periosteum. The masses were measured $1.5 \times 1.5 \mathrm{~cm}$ on frontozygomatic suture lesion and $1.5 \times 1.0 \mathrm{~cm}$ on infraorbital rim lesion (Fig. 5). We also performed exploration of anterior wall of the maxilla through the previous gingivobuccal incision site to detect any possible simultaneous problems. However, there was no specific finding except clean whitish granuloma-like mass around the previous fixation site of bioabsorbable plate on the maxilla. All the specimens were sent for biopsy and revealed foreign body granuloma as well. After the en-bloc excision of all the lesions, the patient recovered completely with subsided signs and symptoms at 6 months postoperatively (Fig. 6).

\section{DISCUSSION}

Various bioabsorbable materials have been developed to overcome the disadvantages of metal implants such as visibility on radiography, palpability, bone erosion, growth restriction, thermal sensitivity, and the need for removal. Their efficacy and clinical utility have been increasingly reported in the field of hand $[7,8]$, maxillofacial skeleton surgery although it is still controversial for use in the mandibular fixation [1,2]. However, concerns about potential delayed adverse reactions, especially foreign body reaction, have been still raised.

Ideally, bioabsorbable implant should provide a firm fixation in the initial bone healing phase and should be degraded after complete osteosynthesis without any complications. However, there have been some reports associated with unavoidable sec-
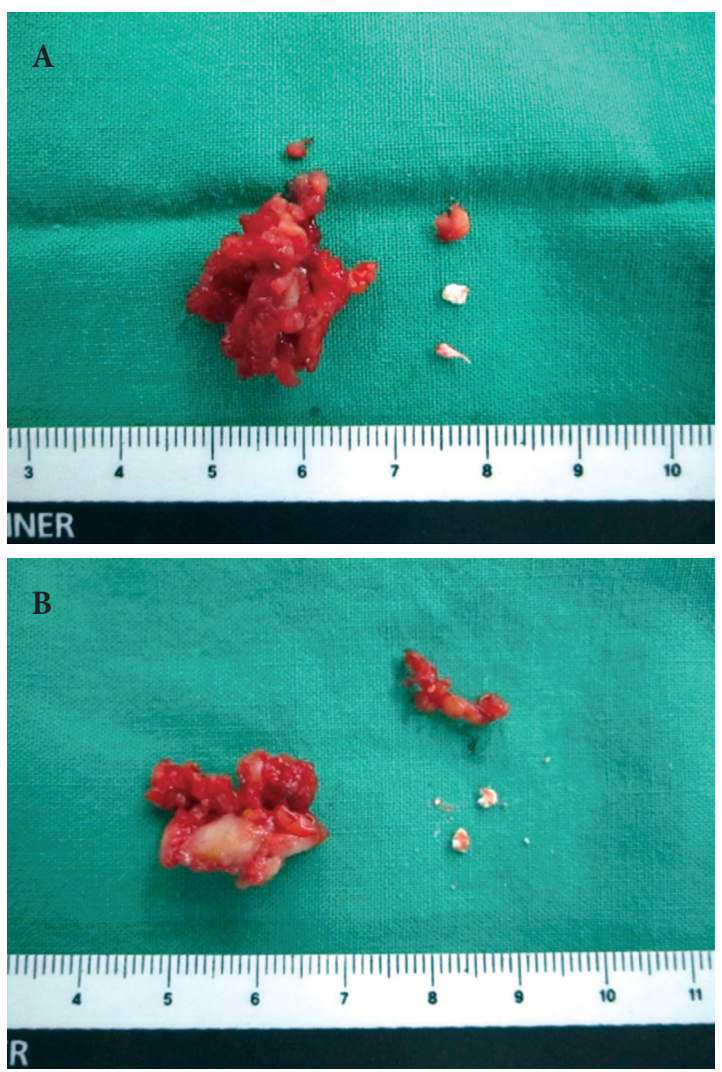

Fig. 5. Specimens from frontozygomatic suture (A) and left inferior orbital wall (B).

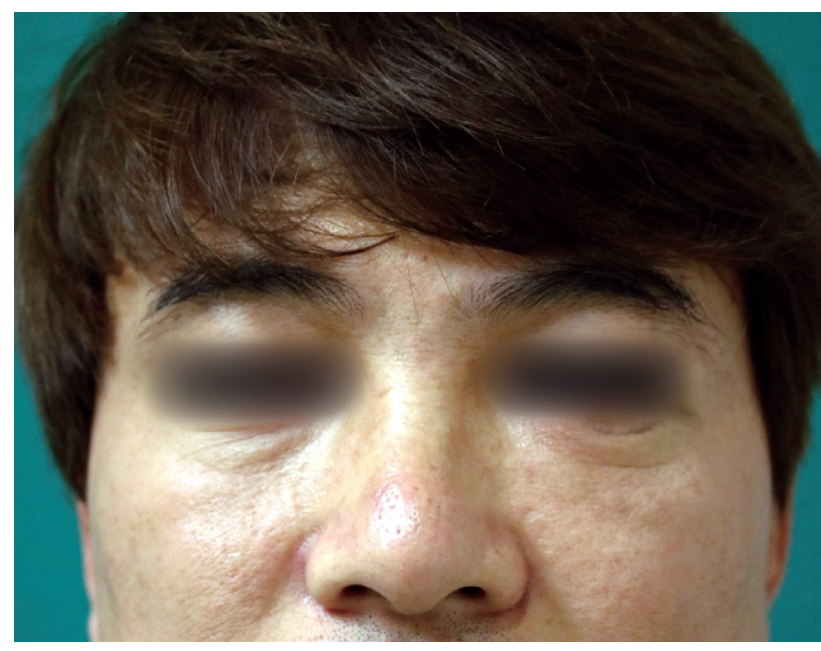

Fig. 6. Postoperative photograph on left lateral eyebrow and periorbital region at 6 months after en-bloc resection of the lesion under general anesthesia.

ondary surgery due to delayed adverse effect even after successful operation. Several studies reported the cases of delayed foreign 
body reaction to bioabsorbable implants in metacarpal bone fracture $[7,8]$.

Furthermore, there have been other reports of complications due to inadequate degradation after maxillofacial surgery associated with bioabsorbable materials. Jeon et al. [1] recently reported eight cases of frontozygomatic suture and infraorbital rim fracture treated with bioabsorbable plates presenting delayed foreign body reaction. They reported that if the plate is larger and thicker, the occurrence rate of delayed foreign body reaction tends to increase.

Bioabsorbable material inside the body might induce a substance-dependent inflammatory reaction such as foreign body reaction. This reaction is divided into five steps: protein adsorption, acute and chronic inflammation, foreign body giant cell formation and fibrosis [13]. When the bioabsorbable polymeric material reaches the final stage of the degradation process, it is known to elicit a local foreign body reaction. Macrophages play an important role in this process, and the large polynucleated cells are formed due to macrophage fusion $[13,14]$. Although the symptoms of this response mostly pass unnoticed, foreign body reaction such as delayed sterile abscess could rarely occur [9].

Multiple factors are believed to affect the secondary surgery for removal of the bioabsorbable implants. Chemical components, size and shape of bioabsorbable plates are known to be associated with the occurrence of delayed foreign body reaction $[9,10]$. In the present study, we suggest that the thickness of soft tissue above the bioabsorbable implant might be another important factor related to the adverse reaction. As the thickness of soft tissue is increased, the less intensity of the stimulus reaches the implant. Then, the degradation process of implant is not disturbed and the possibility of complication will decrease. There have been case reports of this reaction occurring at superficial layer such as frontozygomatic suture and inferior orbital rim fracture $[1,2,15]$ and metacarpal bone fracture $[7,8]$. However, in deep layer such as fracture of anterior wall of maxillary sinus, there have been no reports of delayed foreign body reaction caused by bioabsorbable plates until now. In the same manner, in our case, the delayed sterile abscess occurred in the frontozygomatic suture and the inferior orbital rim region.

Plate degradation properties and rates vary with the nature of the bioabsorbable plates, such as implant volume and chemical composition. It is highly suggestive that external stimulation, which is reduced by the soft tissue above the implant, might affect the degradation process. Inappropriate degradation can cause a delayed foreign body reaction leading to second exploratory operation. Although most bioabsorbable implants are completely degraded in 2-4 years postoperatively [1], it is necessary to consider delayed complications when using this material, and plastic surgeons should notice patients about this probable complication before surgery.

\section{CONFLICT OF INTEREST}

No potential conflict of interest relevant to this article was reported.

\section{PATIENT CONSENT}

The patients provided written informed consent for the publication and the use of their images.

\section{REFERENCES}

1. Jeon HB, Kang DH, Gu JH, Oh SA. Delayed foreign body reaction caused by bioabsorbable plates used for maxillofacial fractures. Arch Plast Surg 2016;43:40-5.

2. Bell RB, Kindsfater CS. The use of biodegradable plates and screws to stabilize facial fractures. J Oral Maxillofac Surg 2006;64:31-9.

3. Wong FK, Adams S, Hudson DA, Ozaki W. Use of resorbable fixation system in pediatric facial fractures. J Craniofac Surg 2017;28:635-7.

4. Tuncer S, Yavuzer R, Kandal S, Demir YH, Ozmen S, Latifoglu O, et al. Reconstruction of traumatic orbital floor fractures with resorbable mesh plate. JCraniofac Surg 2007;18:598-605.

5. Landes C, Hoefer SH, Richards T, Walcher F, Sader R. Perspectives of patients about bioabsorbable internal fixation for maxillofacial fractures. Ann Maxillofac Surg 2015;5:185-90.

6. Wood RJ, Petronio JA, Graupman PC, Shell CD, Gear AJ. New resorbable plate and screw system in pediatric craniofacial surgery. J Craniofac Surg 2012;23:845-9.

7. Givissis PK, Stavridis SI, Papagelopoulos PJ, Antonarakos PD, Christodoulou AG. Delayed foreign-body reaction to absorbable implants in metacarpal fracture treatment. Clin Orthop Relat Res 2010;468:337783.

8. Choi JS, Lee JH, Kim SM, Kim YJ, Jun YJ. Foreign-body granuloma after metacarpal fracture treatment with absorbable implants. Arch 
Plast Surg 2015;42:505-7.

9. Bostman O, Pihlajamaki H. Clinical biocompatibility of biodegradable orthopaedic implants for internal fixation: a review. Biomaterials 2000;21:2615-21.

10. Xue AS, Koshy JC, Weathers WM, Wolfswinkel EM, Kaufman Y, Sharabi SE, et al. Local foreign-body reaction to commercial biodegradable implants: an in vivo animal study. Craniomaxillofac Trauma Reconstr 2014;7:27-34.

11. Middleton JC, Tipton AJ. Synthetic biodegradable polymers as orthopedic devices. Biomaterials 2000;21:2335-46.
12. Konofaos P, Goubran S, Wallace RD. The role of resorbable mesh as a fixation device in craniosynostosis. J Craniofac Surg 2016;27:105-8.

13. Anderson JM, Rodriguez A, Chang DT. Foreign body reaction to biomaterials. Semin Immunol 2008;20:86-100.

14. Klopfleisch R. Macrophage reaction against biomaterials in the mouse model: phenotypes, functions and markers. Acta Biomater 2016;43:313 .

15. Aryasit O, Ng DS, Goh AS, Woo KI, Kim YD. Delayed onset porous polyethylene implant-related inflammation after orbital blowout fracture repair: four case reports. BMC Ophthalmol 2016;16:94. 